\title{
Lamb Meat Quality Assessment by Support Vector Machines ${ }^{\dagger}$
}

\author{
Paulo Cortez (pcortez@dsi.uminho.pt) \\ Department of Information Systems, University of Minho, 4800-058 Guimarães, \\ PORTUGAL
}

Manuel Portelinha (mportelinha@ipb.pt), Sandra Rodrigues (srodrigues@ipb.pt), Vasco Cadavez (vcadavez@ipb.pt) and Alfredo Teixeira (teixeira@ipb.pt)

Bragança Polytechnic Institute, 5301-855 Bragança, PORTUGAL

\begin{abstract}
The correct assessment of meat quality (i.e., to fulfill the consumer's needs) is crucial element within the meat industry. Although there are several factors that affect the perception of taste, tenderness is considered the most important characteristic. In this paper, a Feature Selection procedure, based on a Sensitivity Analysis, is combined with a Support Vector Machine, in order to predict lamb meat tenderness. This real-world problem is defined in terms of two difficult regression tasks, by modeling objective (e.g. Warner-Bratzler Shear force) and subjective (e.g. human taste panel) measurements. In both cases, the proposed solution is competitive when compared with other neural (e.g. Multilayer Perceptron) and Multiple Regression approaches.
\end{abstract}

Keywords: Regression; Multilayer Perceptrons; Support Vector Machines; Meat Quality; Data Mining; Feature Selection

\section{Introduction}

A top priority factor in the success of meat industry relies on the ability to deliver specialties that satisfy the consumer's taste requirements. In particular, assessing the quality of an item is important for lamb meat firms, specially if they want to move into niche markets by differentiating their products. Therefore, meat and animal scientists have dedicated high efforts in finding reliable quality estimators. Among the several factors that influence meat quality (e.g. juiciness, appearance or aroma), tenderness is considered the most important attribute [11]. In effect, consumers are willing to pay premium prices for tender meat.

The ideal method for measuring tenderness should be accurate, fast, automated and noninvasive. In the past, two major approaches have been proposed [1]: instrumental and sensory analysis. The former is based in an objective test, such as the Instron instrument, which measures the Warner-Bratzler Shear (WBS) force and it is the most commonly used device. On the other hand, sensory methods are based

\footnotetext{
$\dagger$ In Neural Processing Letters, Springer, In press, ISSN:1370-4621.
} 
in subjective information, usually given by a human taste panel. Both approaches are invasive, expensive and time demanding, since they require laboratory work. For instance, the $W B S$ values can only be obtained 72 hours after slaughtering, while the preparation and execution of consumer taste panel may take several days.

An alternative is to use cheap and non invasive carcass measurements that can be collected within the first 24 hours after slaughtering (e.g. $\mathrm{pH}$ and color). Under this scenario, the classic animal science approach is based on Multiple Regression models [1], using meat features as independent (or input) variables and the $W B S$ or sensory measures as the depended (or output) ones. However, the linear models will fail when nonlinear relationships are present. In such cases, a better option is to use techniques such as Neural Networks (NNs) [9] or Support Vector Machines (SVMs) [20]. Indeed, these methods are gaining an attention within the Data Mining field, due to their performance in terms of predictive knowledge [16][8]. It should be stressed that $S V M s$ present theoretical advantages over $N N s$, such as the absence of local minima in the model optimization phase.

In Data Mining applications, besides obtaining a high predictive performance, it is often useful to provide explanatory knowledge. In particular, the measure of input importance is relevant within this domain. Since carcass features are often highly correlated, Principal Component Analysis has been proposed to reduce the input dimensionality [1]. Yet, the principal components are compressed variables and they do not represent a direct meaning for the meat user. An alternative, is to use a Sensitivity Analysis procedure, which has outperformed other input selection techniques (e.g. Forward Selection and Genetic Algorithms) [12].

In the last few years, several authors have proposed nonlinear methods to assess meat quality (e.g. beef, pork, poultry or sausages) [1]. In the majority of these studies, the Multilayer Perceptron neural architecture is the most common approach. However, regarding tenderness prediction, the literature seems scarce and it is primarily oriented towards beef. For example, in the work of Li et al. [15], Multilayer Perceptrons outperformed a Multiple Regression when mapping beef texture images with sensory tenderness scores. In another study, Hill et al. [10] have applied Multilayer Perceptrons to predict the Instron force, obtaining better results than the Multiple Regression method. More recently, Diez et el. [6] adapted a $S V M$ with a polynomial kernel of degree 2 to model beef tenderness preferences, surpassing linear and cubic regression methods.

In this work, the combination of a feature selection procedure, based on a Sensitivity Analysis, with a gaussian kernel $S V M$ is proposed to 
predict lamb meat tenderness. This real-world problem will be modeled in terms of two regression tasks, using both instrumental and sensory measurements. The devised strategy will be tested on animal data and compared with other $N N$ and Multiple Regression approaches.

The paper is organized as follows. First, a description is given on the datasets used (Section 2.1). Then, the learning models are presented (Section 2.2). In Section 3, the experiments performed are described and the results analyzed. Finally, closing conclusions are drawn (Section $4)$.

\section{Materials and Methods}

\subsection{Lamb Meat Data}

This study considered lamb animals with the Protected Designation of Origin certificate, from the Trás-os-Montes northeast region of Portugal. The database was collected from November/2002 until November/2003, with each instance denoting the readings obtained from a slaughtered animal. With a total of 81 examples, the database is quite small. However, it should be noted that each animal presents considerable costs, around 6 euros per kilogram plus laboratory work. Table I presents a synopsis of the data attributes. The HCW is obtained one hour after slaughter, exfoliation and evisceration. The former two attributes (Breed and Sex) are also registered at slaughterhouse, while the others are measured in laboratory. Due to their visual nature, the color attributes $\left(\mathbf{a}^{*}, \mathbf{b}^{*}, \mathbf{d} \mathbf{E}, \mathbf{d} \mathbf{L}\right.$ and $\left.\mathbf{d B}^{*}\right)$ have a high impact in consumer's perception. In most of the situations, these are the only attributes that the consumer can judge.

$$
\text { *** Insert Table I around here } * * *
$$

The WBS force is the major index for measuring meat tenderness. It can only be obtained in laboratory, no sooner than 72 hours after slaughter, by using an invasive device called Instron. The WBS registers the force (in $\mathrm{kg}$ ) required to crush a meat sample with a thickness of $1 \mathrm{~cm}$. Low values suggest tender meat while high readings suggest toughness. On the other hand, a more elaborated scheme was devised to obtain the sensory values (STP). A panel of 12 trained individuals, from the Bragança Polytechnic Institute, was selected. Then, meat samples from the longissinus thoracis muscle were collected and defrost at $4^{\circ} \mathrm{C}$ in a refrigerator. Next, each sample was randomly encoded with a 3 digit number, wrapped in an aluminum sheet and heated at $100^{\circ} \mathrm{C}$. Then, each panel member was set in an individual compartment, 
performing a taste proof, under similar conditions, of random selected samples. Between different tastes, mouths were cleaned by using water and by eating small golden apple pieces. Each sample was ranked from 0 (the most tender) to 10 (the most tough). Finally, the $\mathbf{S T P}$ attribute was measured as the average of the grades from the panel.

Since the original data contained missing values ( 2 for the WBS and 10 for the STP), two new datasets were created by discarding these entries. The first contains 79 rows (for the WBS task), while the second has 71 examples (STP). Figure 1 shows the histograms of the target variables.

$$
\text { *** Insert Figure } 1 \text { around here }{ }^{* * *}
$$

\subsection{Learning Models}

A regression dataset $D$ is made up of $k \in\{1, \ldots, N\}$ examples, each mapping an input vector $\left(x_{1}^{k}, \ldots, x_{I}^{k}\right)$ to a given target $y_{k}$. The error is given by: $e_{k}=y_{k}-\widehat{y}_{k}$, where $\widehat{y}_{k}$ represents the predicted value for the $k$ input pattern. The overall performance is computed by a global metric, namely the Mean Absolute Deviation (MAD) and Relative Mean Absolute Deviation (RMAD), which can be computed as [6]:

$$
\begin{aligned}
& M A D=1 / N \times \sum_{i=1}^{N}\left|y_{i}-\widehat{y}_{i}\right| \\
& R M A D=1 / N \times M A D / \sum_{i=1}^{N}\left|y_{i}-\bar{y}_{i}\right| \times 100(\%)
\end{aligned}
$$

In both metrics, lower values result in better predictive models. The $R M A D$ statistic is scale independent, where $100 \%$ denotes an error similar to the naive average predictor $(\bar{y})$.

The Multiple Regression (MR) model is defined by the equation [8]:

$$
\widehat{y}=w_{0}+\sum_{i=1}^{I} w_{i} x_{i}
$$

where $\left(x_{1}, \ldots, x_{I}\right)$ denotes the input vector and $\left\{w_{0}, \ldots, w_{I}\right\}$ the parameters to be adjusted. This model is easy to interpret and has been widely used in regression applications.

This study will consider the Multilayer Perceptron [9], the most popular Neural Network (NN) architecture. The base network will use biases, one hidden layer of $H$ hidden nodes and logistic activation functions and one output node with a linear function [8]. Thus, each regression task (WBS and $\mathbf{S T P}$ ) will be modeled by a different $N N$. The overall model is given by the equation:

$$
\widehat{y}=w_{o, 0}+\sum_{j=I+1}^{o-1} f\left(\sum_{i=1}^{I} x_{i} w_{j, i}+w_{j, 0}\right) w_{o, i}
$$


where $w_{i, j}$ denotes the weight of the connection from node $j$ to $i, o$ the output node and $f$ the logistic function $\left(\frac{1}{1+e^{-x}}\right)$.

The $N N$ performance will be sensitive to the topology choice. To solve this issue, a common practice is to use a large number of hidden nodes $(H)$ and train the $N N$ with a regularization method. Thus, a weight decay procedure will be adopted, where the hyperparameter $\lambda$ will control the network complexity [8].

All attributes are standardized to a zero mean and one standard deviation. Then, the initial neural weights are randomly set within the range $[-0.7,+0.7]$. Next, the training algorithm is applied and stopped when the error slope approaches zero or after a maximum of $E$ epochs. Since the $N N$ cost function is nonconvex (with multiple minima), $R$ runs will be applied to each neural configuration, being selected the $N N$ with the lowest penalized error.

In Support Vector Machine (SVM) regression, the input $x \in \Re^{I}$ is transformed into a high $m$-dimensional feature space, by using a nonlinear mapping. Then, the $S V M$ finds the best linear separating hyperplane in the feature space:

$$
\widehat{y}=w_{0}+\sum_{i=1}^{m} w_{i} \phi_{i}(x)
$$

where $\phi_{i}(x)$ represents a nonlinear transformation, according to the kernel function $K\left(x, x^{\prime}\right)=\sum_{i=1}^{m} \phi_{i}(x) \phi_{i}\left(x^{\prime}\right)$.

To estimate the best $S V M$, the $\epsilon$-insensitive loss function (Figure 2 ) is often used [18]. The Radial Basis Function kernel, which presents less hyperparameters and numerical difficulties than other kernels (e.g. polynomial or sigmoid), will also be adopted [4]:

$$
K\left(x, x^{\prime}\right)=\exp \left(-\gamma\left\|x-x^{\prime}\right\|^{2}\right), \gamma>0
$$

Under this setup, the performance is affected by three parameters: $C$, a trade-off between the model complexity and the amount up to which deviations larger than $\epsilon$ are tolerated; $\epsilon$, the width of the $\epsilon$ insensitive zone; and $\gamma$, the parameter of the kernel. Since the search space for the three parameters is high, the $C$ and $\epsilon$ values will be set using the heuristics proposed in [5]:

$$
\begin{aligned}
C & =3 \sigma_{y}, \text { if } \bar{y}=0 \\
\widehat{\sigma} & =1.5 / N \times \sum_{i=1}^{N}\left(y_{i}-\widehat{y}_{i}\right)^{2} \\
\epsilon & =\widehat{\sigma} / \sqrt{N}
\end{aligned}
$$

where $\sigma_{y}$ denotes the standard deviation of the output $(y)$ and $\widehat{y}$ is the value predicted by the 3 -nearest neighbor algorithm. Since all variables were standardized to a zero mean, the $\bar{y}=0$ condition is met. 
The hyperparameters $(\lambda$ and $\gamma)$ will be tuned by a two level gridsearch. The first level will search the best value $\left(\lambda_{1}\right.$ or $\left.\gamma_{1}\right)$ within the ranges $\lambda \in\{0.00,0.01, \ldots, 0.20\}$ or $\gamma \in\left\{2^{-15}, 2^{-13}, \ldots, 2^{3}\right\}$, as advised in $[8,4]$. The second level proceeds with a fine tune within the range $\lambda_{2} \in\left\{\lambda_{1}-0.005, \ldots, \lambda_{1}-0.001, \lambda_{1}+0.001, \ldots, \lambda_{1}+0.004\right\} \wedge \lambda_{2} \geq$ 0 or $\gamma_{2} \in\left\{2^{s_{1}-1.75}, \ldots, 2^{s_{1}-0.25}, 2^{s_{1}+0.25}, \ldots, 2^{s_{1}+1.25}\right\} \wedge \gamma_{2} \geq 0$. The prediction accuracy $(M A D)$ in the grid-search is estimated by adopting a 10-fold cross-validation [13]. After obtaining the best parameter, the final model is optimized using the whole training data.

\subsection{Feature Selection}

Nonlinear models such as $N N s$ and $S V M s$ are sensitive to the curse of dimensionality [8][2], i.e., the number of samples should grow exponentially as number of inputs increases. Hence, when small datasets are available, feature selection is expected to reduce the prediction error. Moreover, measurement requirements are reduced and simpler models, which are easier to interpret by the final user, are produced.

In this work, a Sensitivity Analysis procedure will be used to guide the feature selection search. The Sensitivity Analysis is performed after model estimation and it is measured by the variance $\left(V_{a}\right)$ produced in the output $(\widehat{y})$ when the input attribute $(a)$ is moved through its entire range [12]:

$$
\begin{aligned}
& V_{a}=\sum_{i=1}^{L}\left(\widehat{y}_{i}-\overline{\hat{y}}\right) /(L-1) \\
& R_{a}=V_{a} / \sum_{j=1}^{I} V_{j} \times 100(\%)
\end{aligned}
$$

where $I$ denotes the number of input attributes and $R_{a}$ the relative importance of the $a$ attribute. The $\widehat{y}_{i}$ output is obtained by holding all input variables at their average values. The exception is $x_{a}$, which varies through its entire range with $L$ levels.

The proposed feature selection will work as an iterative backward method, using all inputs at the beginning. In each iteration, a 10-fold cross-validation is performed over the training data. The intention is to get a robust estimation of the quality of the inputs. Thus, the input importance values $\left(R_{a}\right)$ are averaged over the 10 -fold trainings and the least important attribute $\left(\bar{R}_{\text {min }}\right)$ is discarded. Due to the computational effort, only one hyperparameter, set to the middle of the first level search range $\left(\lambda=0.1\right.$ or $\left.\gamma=2^{-7}\right)$, is tested during this phase. The algorithm is stopped after $T$ iterations. Then, the second level cross-validation grid search is executed, in order to fine tune the hyperparameter. Finally, the best model is trained using the whole training data. 


\section{Results}

All experiments were conducted with a Pentium $I V$ processor, under the Linux operating system. The simulations were programmed in the $\mathbf{R}$ environment [17], an open source and high-level programming language that provides powerful tools for statistical analysis. The NNs were trained with the $B F G S$ algorithm from the family of quasi-Newton methods, as implemented in the $\mathbf{R}$ nnet library. The $\mathbf{R}$ kernlab package was adopted for the $S V M$ fitting, which uses the Sequential Minimal Optimization algorithm that is implemented by the LIBSVM tool [4].

After preliminary experiments, the maximum number of $N N$ training epochs was set to $E=10$. Further values increased the computational effort with no improvement in performance. The number of hidden nodes was fixed to $H=12$ and the number of runs was set to $R=3$. Regarding the $S V M s$, the tolerance of termination criterion was set to the default value (0.001). Finally, the sensitivity parameters were set to $L=2$ for the binary attributes and $L=5$ for the continuous inputs, while the termination criterion was set to $T=6$. This last value was set after monitoring the validation error progress in some of the initial experiments.

In order to compare the learning models, 30 runs of a leave-oneout procedure [13] (computed over all available data) were executed (in a total of $30 \times N$ experiments). The results are shown in Table II, in terms of the average of the test errors, with the correspondent t-student $95 \%$ confidence intervals [7]. Column Time denotes the required computation for each method (in seconds).

$$
\text { *** Insert Table II around here } * * *
$$

First, the analysis will be given for the models what use all twelve inputs. The Multiple Regression (MR) results are the worst for the WBS task. This scenario changes for the sensory panel, where the $M R$ is the second best method, outperforming the $N N$ method. Regarding the nonlinear methods, the $S V M$ is the best method for both datasets, outperforming (with statistical significance) the $N N$ and $M R$ models. In addition, the computational effort also favors the $S V M$, since the $N N$ demands a computational increase around a factor of 2.5. Overall, the $R M A D$ values suggest that the second task is more difficult than the first one.

While only using half the inputs, the Feature Selection (FS) based approaches (FSNN and FSSVM) give rise to better/slightly better performances. In terms of the average $R M A D$ values and for the WBS output, there is an improvement of $0.6 \%$ (not statisticaly significant) 
for the FSNN and $2.0 \%$ (statistically significant) for the FSSVM model. Turning to the second task, the average $R M A D$ decrease is $7.3 \%$ (statistically significant) for the $F S N N$ and $0.2 \%$ (not statistically significant) for the FSSVM. In terms of the final comparison, FSSVM is the advised method, since it presents a lower mean and confidence interval values, when compared with the other models.

Table III shows the average relative importance (Equation 7 ) of the input variables for the best methods. To simplify the analysis, the less important attributes $\left(R_{a} \leq 1 \%\right)$ were removed from the table (Sex, $\mathbf{C}$ and $\left.\mathbf{b}^{*}\right)$. It should also be noted that the table contains more than six attributes, since in each simulation different sets of features can be selected. The Sex attribute is the least relevant factor $\left(R_{a} \leq 0.1 \%\right)$, which contrasts with the knowledge that gender affects tenderness. Since female meat often present a higher weight and fatness, the sex information could be indirectly represented in the HCW and STF2 variables. However, additional experiments where these attributes were replaced by the Sex input and the models retrained did not provide evidence for this claim.

*** Insert Table III around here ***

In general, the results are similar for both $N N$ and $S V M$ based methods. For the WBS task, the red color $\left(\mathbf{a}^{*}\right)$ is the highest important attribute. Turning to the STP problem, the most relevant features are the Breed and red index $\left(\mathbf{a}^{*}\right)$. The differences obtained between the two tasks may be explained by psychological factors. For instance, the Breed importance increased from 0.4/0.3\% (WBS) to $35.3 / 41.3 \%$ (STP). This is a surprising result, since it contradicts the animal science theory.

As an example, the left of Figure 3 shows the scatter plots (predicted versus the observed values) for the WBS task. In the figure, the diagonal line denotes the perfect forecast. The Regression Error Characteristic $(R E C)$ curves [3] are also shown (right of the figure) for the FSSVM, MR and Average Predictor methods. The REC curve is used to compare regression models and it plots the error tolerance $(x-$ axis), given in terms of the absolute deviation, versus the percentage of points predicted within the tolerance ( $y$-axis). In the figure, the FSSVM line is above the other curves for the majority of the $x$ values. Overall, it presents an higher area, denoting a better fit.

\footnotetext{
*** Insert Figure 3 around here $* * *$
} 


\section{Conclusions}

In this work, a Feature Selection (FS) procedure, based on a Sensitivity Analysis, is combined with a Support Vector Machine (SVM), aiming at the prediction of lamb meat tenderness. This real-world problem was addressed by two distinct regression tasks by using instrumental and sensory measurements. The former is based in the Warner-Bratzler Shear $(W B S)$ force, which is an objective measure obtained from a special device called Instron. The latter involves the use of subjective information, requiring the execution of a human Sensory Taste Panel (STP). In both cases, the FSSVM combination outperformed other Neural Network (NN) and Multiple Regression configurations.

The final solution is much simpler, requiring only half the number of inputs (6 instead of 12). Moreover, the proposed method is noninvasive, much cheaper than the $W B S$ or $S T P$ procedures, and can be computed just $1(S T P)$ or 24 hours $(W B S)$ after slaughter. This opens the room for the development of automatic tools for decision support [19].

One drawback may be the obtained accuracy, which is only $18 \%$ $(W B S)$ and $12 \%(S T P)$ better than the simple average predictor. However, it should be stressed that the tested datasets are very small. As argued by Lavrăc et al. [14], there are important Data Mining applications where the data is scarce and more research is needed towards methods that can deal with such datasets. This work backs this claim. Furthermore, Díez et al. [6] considered the modeling of sensory preferences a very difficult regression task. To our knowledge, this is the first time lamb meat quality is approached by $S V M s$ and further exploratory research needs to be performed.

Another relevant point regards the input importance. Some results, such as the gender null impact and breed relevance (for the STP task), seem to contradict the animal science theory. Regarding the breed importance, the results were discussed with the experts, which then discovered that the Mirandesa lambs were considered less stringy and more odor intense, which may be due to animal stress during slaughter. Nevertheless, further research is needed towards this issue.

In future work, the proposed approach will be tested in a real environment, by attaching computer systems with friendly human interfaces into meat laboratories and/or slaughterhouses. This will allow us to obtain, after some time, a valuable feedback from the meat users, and also to enrich the datasets by gathering more meat samples. 


\section{References}

1. I. Arvanitoyannis and M. Houwelingen-Koukaliaroglou. Implementation of Chemometrics for Quality Control and Authentication of Meat and Meat Products. Critical Reviews in Food Science and Nutrition, 43(2):173-218, 2003.

2. Y. Bengio, O. Delalleau, and N. Le Roux. The curse of dimensionality for local kernel machines. Technical Report 1258, Department IRO, Universitsité de Montréal, Canada, May 2005.

3. J. Bi and K. Bennett. Regression Error Characteristic curves. In Proceedings of 20th Int. Conf. on Machine Learning (ICML), Washington DC, USA, 2003.

4. C. Chang C. Hsu and C. Lin. A Practical Guide to Support Vector Classification. http://www.csie.ntu.edu.tw/ cjlin/papers/guide/guide.pdf, July, Dep. of Comp. Science and Information Eng., National Taiwan University, 2003.

5. V. Cherkassy and Y. Ma. Practical Selection of SVM Parameters and Noise Estimation for SVM Regression. Neural Networks, 17(1):113-126, 2004.

6. J. Diéz, G. Bayón, J. Quevedo, J. Coz, O. Luaces, J. Alonso, and A. Bahamonde. Discovering relevancies in very difficult regression problems: applications to sensory data analysis. In Proc. of ECAI, pages 993-994, 2004.

7. A. Flexer. Statistical evaluation of neural networks experiments: Minimum requirements and current practice. In Proc. of the 13th European Meeting on Cybernetics and Systems Research, volume 2, pages 1005-1008, 1996.

8. T. Hastie, R. Tibshirani, and J. Friedman. The Elements of Statistical Learning: Data Mining, Inference, and Prediction. Springer-Verlag, NY, USA, 2001.

9. S. Haykin. Neural Networks - A Compreensive Foundation. Prentice-Hall, New Jersey, 2nd edition, 1999.

10. B. Hill, S. Jones, W. Robertson, and I. Major. Neural Network Modeling of Carcass Measurements to Predict Beef Tenderness. Canadian Journal of Animal Science, (80):311-318, 2000.

11. K. Huffman, M. Miller, L. Hoover, C. Wu, H. Brittin, and C. Ramsey. Effect of beef tenderness on consumer satisfaction with steaks consumed in the home and restaurant. Journal of Animal Sc., 74(1):91-97, 1997.

12. R. Kewley, M. Embrechts, and C. Breneman. Data Strip Mining for the Virtual Design of Pharmaceuticals with Neural Networks. IEEE Transactions on Neural Networks, 11(3):668-679, May 2000.

13. R. Kohavi. A Study of Cross-Validation and Bootstrap for Accuracy Estimation and Model Selection. In Proc. of the Int. Joint Conf. on Artificial Intelligence (IJCAI), Montreal, Canada, August 1995.

14. N. Lavrac, H. Motoda, T. Fawcett, P. Langley, and P. Adriaans. Introduction: Lessons learned from data mining applications and collaborative problem solving. Machine Learning, 57(1-2):13-32, 2004.

15. J. Li, J. Tan, F. Martz, and H. Heymann. Image texture features as indicators of beef tenderness. Meat Science, 53(1):17-22, 1999.

16. S. Mitra, S. Pal, and P. Mitra. Data Mining in Soft Computing Framework: A Survey. IEEE Transactions on Neural Networks, 13(1):3-14, January 2002.

17. R Development Core Team. $R: A$ Language and Environment for Statistical Computing. R Foundation for Statistical Computing, Vienna, Austria, http://www.R-project.org, 2005. ISBN 3-900051-07-0.

18. A. Smola and B. Scholkopf. A tutorial on support vector regression. Technical Report NC2-TR-1998-030, University of London, UK, 1998. 
19. E. Turban, J. Aronson, and T. Liang. Decision Support Systems and Intelligent Systems. Prentice-Hall, 2004.

20. V. Vapnik. The Nature of Satistical Learning Theory. Springer, 2nd edition, 1999.
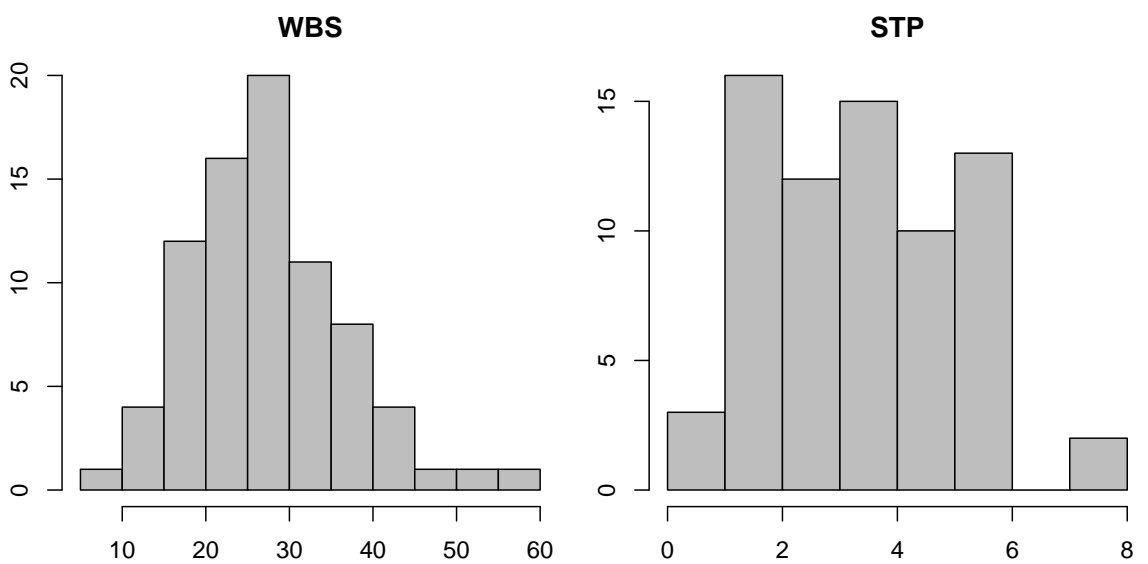

Figure 1. The histograms for the instrumental and sensory output variables

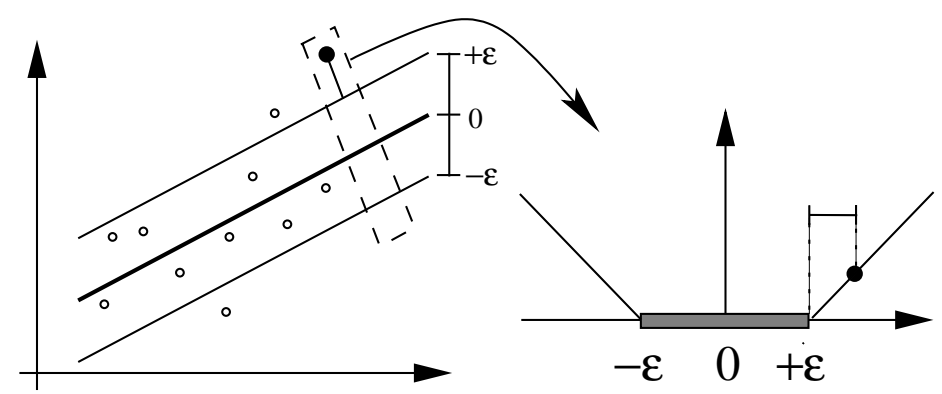

Figure 2. Example of a linear Support Vector Machine regression and the $\epsilon$-insensitive loss function (adapted from [18]) 

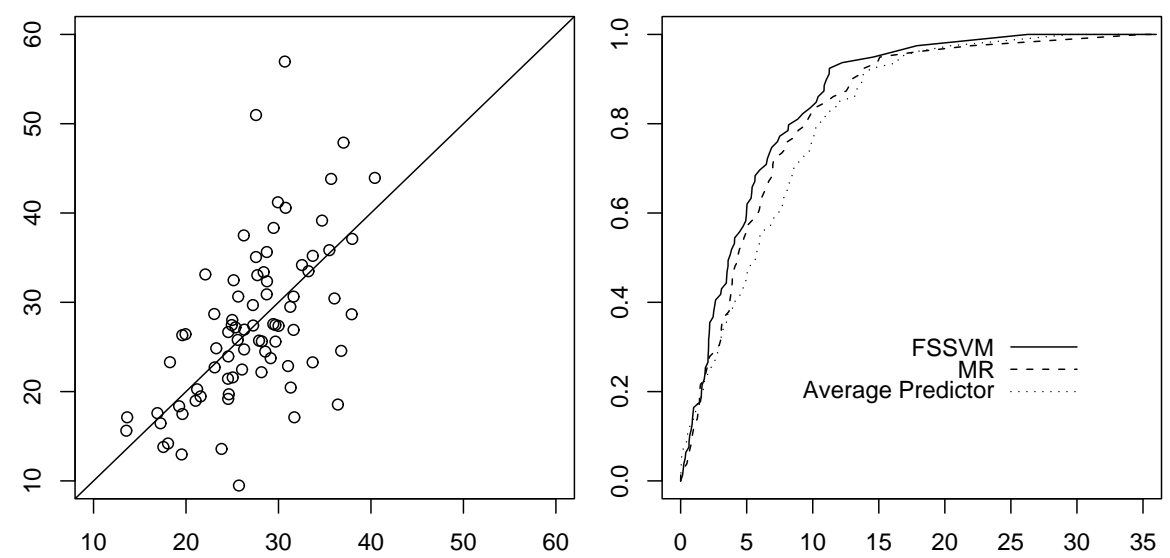

Figure 3. The predicted ( $x$-axis) versus observed (y-axis) values for FSSVM (left) and the REC curves (right) for the WBS task

Table I. The dataset main attributes

\begin{tabular}{lll}
\hline Attribute & Description & Domain \\
\hline Breed & Breed type & $\{$ Bragançana, Mirandesa $\}$ \\
Sex & Lamb sex & $\{$ Male, Female $\}$ \\
HCW & Hot carcass weight $(k g)$ & {$[4.1,14.8]$} \\
STF2 & Sternal fat thickness & {$[6.0,27.8]$} \\
C & Subcutaneous fat depth & {$[0.3,5.1]$} \\
pH1 & pH 1 hour after slaughtering & {$[5.5,6.8]$} \\
pH24 & pH 24 hours after slaughtering & {$[5.5,5.9]$} \\
$\mathbf{a *}$ & Red color index & {$[11.5,22.2]$} \\
$\mathbf{b *}$ & Yellow color index & {$[6.5,12.5]$} \\
dE & Total color difference & {$[46.5,60.9]$} \\
dL & Luminosity differential & {$[-56,-39]$} \\
dB* & Yellow differential & {$[15.3,22.5]$} \\
\hline WBS & Warner-Bratzler Shear force & {$[9.5,57.0]$} \\
STP & Sensory Taste Panel & {$[0.7,7.1]$} \\
\hline
\end{tabular}


Table II. The regression results

\begin{tabular}{llrrrr}
\hline Task & Model & Inputs & Time & $M A D$ & $R M A D$ \\
\hline \multirow{4}{*}{ WBS } & $M R$ & 12 & 53 & $6.22 \pm 0.00$ & $91.42 \pm 0.00$ \\
& $N N$ & 12 & 69869 & $6.17 \pm 0.09$ & $90.56 \pm 1.27$ \\
& $S V M^{\star}$ & 12 & 28202 & $5.73 \pm 0.04$ & $84.16 \pm 0.52$ \\
& $F S N N$ & 6 & 72698 & $6.12 \pm 0.06$ & $89.94 \pm 0.81$ \\
& $F S S V M^{\dagger} \diamond$ & 6 & 60554 & $\mathbf{5 . 6 0} \pm 0.02$ & $\mathbf{8 2 . 1 8} \pm 0.33$ \\
\hline \multirow{6}{*}{ STP } & $M R$ & 12 & 46 & $1.24 \pm 0.00$ & $90.31 \pm 0.00$ \\
& $N N$ & 12 & 60512 & $1.35 \pm 0.02$ & $98.21 \pm 1.19$ \\
& $S V M^{\star}$ & 12 & 24536 & $1.22 \pm 0.01$ & $88.48 \pm 0.83$ \\
& $F S N N^{\dagger}$ & 6 & 63345 & $1.25 \pm 0.02$ & $90.91 \pm 1.16$ \\
& $F S S V M^{\diamond}$ & 6 & 52952 & $\mathbf{1 . 2 1} \pm 0.01$ & $\mathbf{8 8 . 2 8} \pm 0.40$ \\
\hline
\end{tabular}

$\star$ - $\quad$ Statistically significant $(p$-value $<0.05)$ under pairwise comparisons with the previous $M R$ and $N N$ models

$\dagger$ - Statistically significant under a pairwise comparison with the same model without the $F S$ procedure

$\diamond$ - Statistically significant under a pairwise comparison with FSNN

Table III. The relative importance of the input variables (in \%)

\begin{tabular}{llrrrrrrrrr}
\hline \multirow{2}{*}{ Task } & \multirow{2}{*}{ Model } & \multicolumn{1}{c}{ Attribute } \\
& & Bre. & HCW & STF2 & pH1 & pH24 & $\mathrm{a}^{*}$ & $\mathrm{dE}$ & $\mathrm{dL}$ & $\mathrm{dB}^{*}$ \\
\hline \multirow{2}{*}{ WBS } & $F S N N$ & 0.4 & 7.4 & 5.2 & 0.3 & 1.3 & 58.4 & 20.2 & 2.9 & 3.6 \\
& $F S S V M$ & 0.3 & - & 25.4 & 0.4 & 7.1 & 32.4 & - & 19.2 & 14.9 \\
\multirow{2}{*}{ STP } & $F S N N$ & 35.3 & 2.7 & 4.6 & 12.9 & - & 25.1 & 17.5 & 0.3 & 0.3 \\
& $F S S V M$ & 41.3 & 7.8 & 0.7 & 16.0 & - & 26.3 & - & 0.3 & 6.9 \\
\hline
\end{tabular}


lamb3.tex; 28/04/2006; 14:55; p.14 